\title{
Analytical solution for solute transport from a pulse point source along a medium having concave/convex spatial dispersivity within fractal and Euclidean framework
}

\author{
Vinod Kumar Bharati ${ }^{1} *$, Vijay P Singh ${ }^{2,3}$, Abhishek Sanskrityayn ${ }^{1}$ \\ and NAVEen Kumar ${ }^{1}$ \\ ${ }^{1}$ Department of Mathematics, Banaras Hindu University, Varanasi 221005, India. \\ ${ }^{2}$ Department of Biological Engineering and Agricultural Engineering, Texas A $\dot{\xi} M$ University, \\ College Station, TX, USA. \\ ${ }^{3}$ Zachry Department of Civil Engineering, Texas A\&M University, College Station, TX, USA. \\ ${ }^{*}$ Corresponding author. e-mail: vinodkmr418@gmail.com
}

MS received 21 December 2017; revised 4 May 2019; accepted 24 May 2019

In the present study, analytical solutions of the advection dispersion equation (ADE) with spatially dependent concave and convex dispersivity are obtained within the fractal and the Euclidean frameworks by using the extended Fourier series method. The dispersion coefficient is considered to be proportional to the $n$th power of a non-homogeneous quadratic spatial function, where the index $n$ is considered to vary between 0 and 1.5 so that the spatial dependence of dispersivity remains within the limit to describe the heterogeneity in the fractal framework. Real values like $n=0.5$ and 1.5 are considered to delineate heterogeneity of the aquifer in the fractal framework, whereas integral values like $n=1$ represent the same in the Euclidean sense. A concave or convex variation is free from demanding a limiting value as in the case of linear variation, hence it is more appropriate in the ambience of many disciplines in which $\mathrm{ADE}$ is used. In this study, concentration at the source site remains uniform until the source is present and becomes zero once it is annihilated forever. The analytical solutions, validated through the respective numerical solutions, are obtained in the form of an extended Fourier series with only first five terms. They are convergent to the desired concentration pattern and are stable with the Peclet number. It has been possible because of the formulation of a new Sturm-Liouville problem with advective information. The analytical solutions obtained in this paper are novel.

Keywords. Extended Fourier series method (EFSM); Sturm-Liouville problem (SLP); initial value problem (IVP); fractal framework; Euclidean framework.

\section{List of symbols}

$a_{1} \quad$ heterogeneity parameter $(\mathrm{L})$

$a_{2}$ heterogeneity parameter $\left(\mathrm{L}^{-2}\right)$

$b_{0}$ constant

$b_{1}$ non-dimensional heterogeneity parameter $\left(=a_{1} \ell\right)$ $b_{2} \quad$ non-dimensional heterogeneity parameter $\left(=a_{2} \ell^{2}\right)$

$c \quad$ concentration variable $\left(\mathrm{ML}^{-3}\right)$

$c_{0}$ reference concentration parameter $\left(\mathrm{ML}^{-3}\right)$

$C$ non-dimensional concentration variable

$C^{*}$ non-dimensional concentration variable 
$D_{0} \quad$ uniform dispersion coefficient in homogeneous medium $\left(\mathrm{L}^{2} / \mathrm{T}\right)$

$D$ variable dispersion coefficient in heterogeneous medium $\left(\mathrm{L}^{2} / \mathrm{T}\right)$

$\boldsymbol{D}$ diagonal matrix of order $m \times p$

I identity matrix of order $m \times p$

$\ell \quad$ length of domain $(\mathrm{L})$

$N \quad$ number of terms in the EFS

$n$ index of the quadratic expression in equation (3)

$P e \quad$ Peclet number

$\boldsymbol{Q} \quad$ matrix of order $m \times p$

$\boldsymbol{R} \quad$ matrix of order $m \times p$

$\boldsymbol{R}_{1} \quad$ matrix of eigenvectors of order $m \times p$

$s \quad$ Laplace variable

$t$ time variable

$t_{0}$ time when the source has been removed

$T$ non-dimensional time variable

$T_{0}$ non-dimensional source removal time

$u \quad$ variable velocity in heterogeneous medium

$u_{0}$ uniform velocity in homogeneous medium $(\mathrm{L} / \mathrm{T})$

$x$ position variable

$X$ non-dimensional position variable

$\beta_{m}$ time-dependent coefficients of EFS

$\psi_{m}$ orthogonal eigenfunctions of SLP

$\phi_{m}$ orthonormal eigenfunctions of SLP

$\lambda_{m}$ eigenvalues of SLP

$v_{m} \quad$ eigenvalues of $\boldsymbol{B}$

$\alpha_{m}$ roots of transcendental equation

\section{Introduction}

Advection dispersion equation (ADE) is a partial differential equation (PDE) of the parabolic type derived on the principle of conservation of mass. Apart from the study of transport of solute mass originating from the pollutant's source and being released in the environmental domain of air, soil, groundwater and rivers, ADE is widely used to elaborate (i) flow in packed columns involving chemical reactions or separation of chemical components; pore diffusion of gases, chromatography, ion exchange (Hiester and Vermeulen 1952; Houghton et al. 1988); (ii) displacement of oil with gas, petroleum and natural gas production (Todd 1989); (iii) conservation of gas such as fumigant being advected and dispersed through a bulk of stored grains under the circumstances when velocity of air through the grains is the superficial, Darcian or phase velocity (Thorpe et al. 1991;
Thorpe and Whitaker 1992); and (iv) migration of cells, growth factors, angiogenesis, during wound healing and controlled release of nutrients from coated spherical fertiliser granules for its optimal uptake by plants and crops (Dale et al. 1996; Cussler 1997; Schugart et al. 2008; Basu et al. 2010). The medium through which mass transport takes place in all such models is porous and seldom homogeneous. Solute transport through a homogeneous medium is represented by an ADE with constant coefficients. The present study enucleates to obtain the analytical solutions of the ADE which are novel in more than one aspect in the perspective of solute mass transport along groundwater. This methodology may be easily used in similar problems of other disciplines stated above.

Different theories have been established experimentally and theoretically to deal with the solute transport along groundwater under real hydrogeological conditions. The assimilation of the heterogeneity in an efficacious way by considering the coefficient(s) of the ADE variable has been proposed. Groundwater velocity is governed by the Darcy equation, according to which it is proportional to hydraulic conductivity and hydraulic gradient. Hydraulic conductivity is seldom uniform. The hydraulic conductivity varies spatially in extensively large heterogeneous aquifers. But experimentalists in hydrology consider the groundwater velocity to be uniform. So spatially dependent dispersivity (the ratio of the dispersion coefficient and velocity), emanating the heterogeneity of the medium is equivalent to the spatially dependent dispersion coefficient. Dispersivity is a key feature influencing the solute transport along the groundwater flow. An observation has been made that in large subsurface formations dispersivity along uniform flow might vary with position or with time (Metheron and de Marsily 1980). Simultaneously, the variation of dispersivity with position was shown in Pickens and Grisak (1981) and the ADE with a linearly dependent dispersion coefficient and uniform velocity was solved using a finite element method. In the same work, to obviate the difficulty of considering the asymptotic limiting value of a linearly increasing spatial function and an exponential form, a quadratic spatially dependent dispersivity was suggested. Gelhar et al. (1992) achieved the scale dependence of dispersivity to discuss solute transport through porous media but their study was not in favour of a single universal law expecting a family of curves reflecting different dispersivities in aquifers. But 
attempts continued to identify a unique scaling law (Neuman 1990; Di Federico and Neuman 1998). The studies which did not support a unique scaling law have been cited in Zech et al. (2015), although this claim has been again readdressed in Neuman (2016).

Analytical solutions of the ADE in one, two and three dimensions were derived in semi-infinite media for describing the solute transport in heterogeneous media with a linearly increasing distance-dependent dispersion coefficient and uniform/steady/unsteady velocity (Yates 1990; Huang et al. 1996; Hunt 1998, 2002; Pang and Hunt 2001; Chen et al. 2008; Gao et al. 2010). Kumar et al. (2009) and Yadav et al. (2011) have studied the one-dimensional solute through heterogeneous media originating from the uniform continuous source and pulse-type source, respectively, by using spatial- and temporal-dependent coefficients of the ADE. You and Zhan (2013) derived the two semi-analytical solutions for solute transport in a finite column with linear asymptotic and exponential distance-dependent dispersivities and timedependent sources along a uniform flow. Analytical solutions for the one-dimensional advection-dispersion equations have been obtained using the Laplace transform technique into the left and right domains at the origin of the river domain (Wadi et al. 2014). In these studies, analytical methods based on Laplace, Fourier, Hankel integral transforms and separation of variables have been used.

But these methods become insufficient in the case of ADE with quadratic non-homogeneous spatial coefficients. As cited above, such form of dispersivity is of immense importance (Pickens and Grisak 1981). Theoretical calculations were carried out in Molz et al. (1983) which showed that the dispersivity attains a uniform value after a travel distance of 5-500 km; however, in Sudicky et al. (1983), this travel distance is suggested to be shorter. Due to the temporal variability in the hydraulic gradient, the derivations in Kinzelbach and Ackerer (1986) lead to decreased apparent longitudinal dispersivity in most cases, whereas the work of Rehfeldt and Gelhar (1993) indicates only enhancement in the dispersion. These findings suggest that the dispersivity increases from a value at the initial end to some higher value at an intermediate position from where it decreases to the same initial value at the other end of a certain length, or follows a reverse trend of variability in that length. The former trend of variation is concave type and the reverse trend is convex type.
Mathematically, concave or convex spatial variation may be described by a quadratic or cubic polynomial. Linear or exponential spatial variation demands asymptotic conditions hence is not suitable to delineate the heterogeneity of the aquifer domain of sufficiently large length.

Now such analytical solutions have been possible using the extended Fourier series method (EFSM), as demonstrated in the present study. The concept involved in EFSM (Haberman 1987) is simple and may be used to find a solution to a problem associated with an ordinary differential equation (ODE) or PDE or integral equation of the Fredholm type, in the form of a Fourier series with constant coefficients (in the case of ODE and integral equation) or extended Fourier series (EFS) with time-dependent coefficients (in the case of a PDE). The EFS is written in terms of orthogonal functions which are non-zero solutions of an eigenvalue problem with a self-adjoint operator, famously known as Sturm-Liouville problem (SLP). The EFS reduces the ADE with an initial condition into a system of first-order ODEs in timedependent coefficients with as many initial conditions, referred to as the system of initial value problems (IVPs). In the past 30 years, this method has been used by another name GITT (generalised integral transformation technique). ADEs with constant, linear and quadratic spatial coefficients, have been solved using GITT (Cotta 1990, 1993; Liu et al. 2000; Cotta et al. 2003, 2010; Cassol et al. 2009; Guerrero et al. 2009, 2013; Castellöes et al. 2010; Chen et al. 2011; Qiu et al. 2011). In all the studies cited above, and others not cited in this paper, but referred in the cited studies, the system of IVP could only be solved numerically because the EFS used to converge to the expected results with a large number of terms prohibiting to have an analytical solution. It varied from 30 to around 1800 terms. The convergence also relied upon the spatial form of dependence and the Peclet number. Also the number of terms required for the convergence varied significantly in the studies using different types of boundary conditions. In these studies, the boundary condition at the origin and that at the far end of the finite domain were considered of the Dirichlet type (solution or first type) or of the Dirichlet type and Neumann type (flux or second type), respectively, or of the Sturm type (mixed or the third type) and the Neumann type, respectively. Another common factor in these papers is the use of the same ODE without the advective term associated in all the respective 
SLPs. Had the series been convergent with only the first few terms like five, six or ten, then the system of IVP would have been solved analytically to have the solution in the analytical algebraic expression form.

A fractal concept of the scale dependency of dispersivity in porous media was developed to quantify heterogeneity (Adler 1985; Wheatcraft and Tyler 1988). It was conceptualised to accommodate the irregular path traversed by a solute particle between two positions at a large distance, which is a supposed smooth path in the Euclidean geometry. This irregular path is similar to that obtained by joining the points at which the water meets the sand along a coastline. Such paths are of the concave or convex shape. In the former work, the scale dependency of dispersivity was described with the fractal power of the position variable, with the power lying between 0 and 1 , whereas in the latter work, this power is stated to occur between 1 and 3. The present study conceptualises these aspects and enunciates the analytical solution of the ADE describing the solute transport pattern influenced by the spatial dispersivity in the fractal and Euclidean framework in the groundwater flow domain. Velocity is considered uniform as stated above in the second paragraph. The dispersion coefficient is considered to be proportional to the $n$th power of a non-homogeneous quadratic polynomial in position variable, where $0<n \leq 1.5$. Three values of the index are taken as $n=0.5,1.0$ and 1.5. In this way, the scale dependency of dispersivity remains within the limit to describe the heterogeneity in the fractal framework stated in the preceding paragraph. Also, due to quadratic function, the spatial variation is either of a concave nature or of convex nature. The solution is obtained in the form of an EFS with time-dependent coefficients in terms of orthogonal functions. These orthogonal functions are non-trivial, infinitely many solutions of an SLP. The ODE associated with the SLP is developed with an advective term, which is different from that used in all the previous studies using this method with a different name GITT (as cited in the preceding paragraph). The selection of the new type SLP has paved the way to have the analytical solution, because the Fourier series thus formed with only the first five terms converges to the desired concentration attenuation pattern, precisely. The source of pollutants mass dispersing in the finite domain is assumed to be uniform and continuous but of pulse nature. In the presence of the source, the concentration at the location of the source remains uniform (see figures 1a, 2a and 3a) and becomes zero as soon as the source is removed forever (see figures $1 b, 2 b$ and $3 b$ ). The tracer concentration at a particular location in the groundwater domain coming to zero once its injection is complete is an example. The same methodology has been used by the authors in their previous studies (Bharati et al. 2017,2018 ) in which velocity was considered a nonhomogeneous linear spatial function and the dispersion coefficient was assumed to be proportional
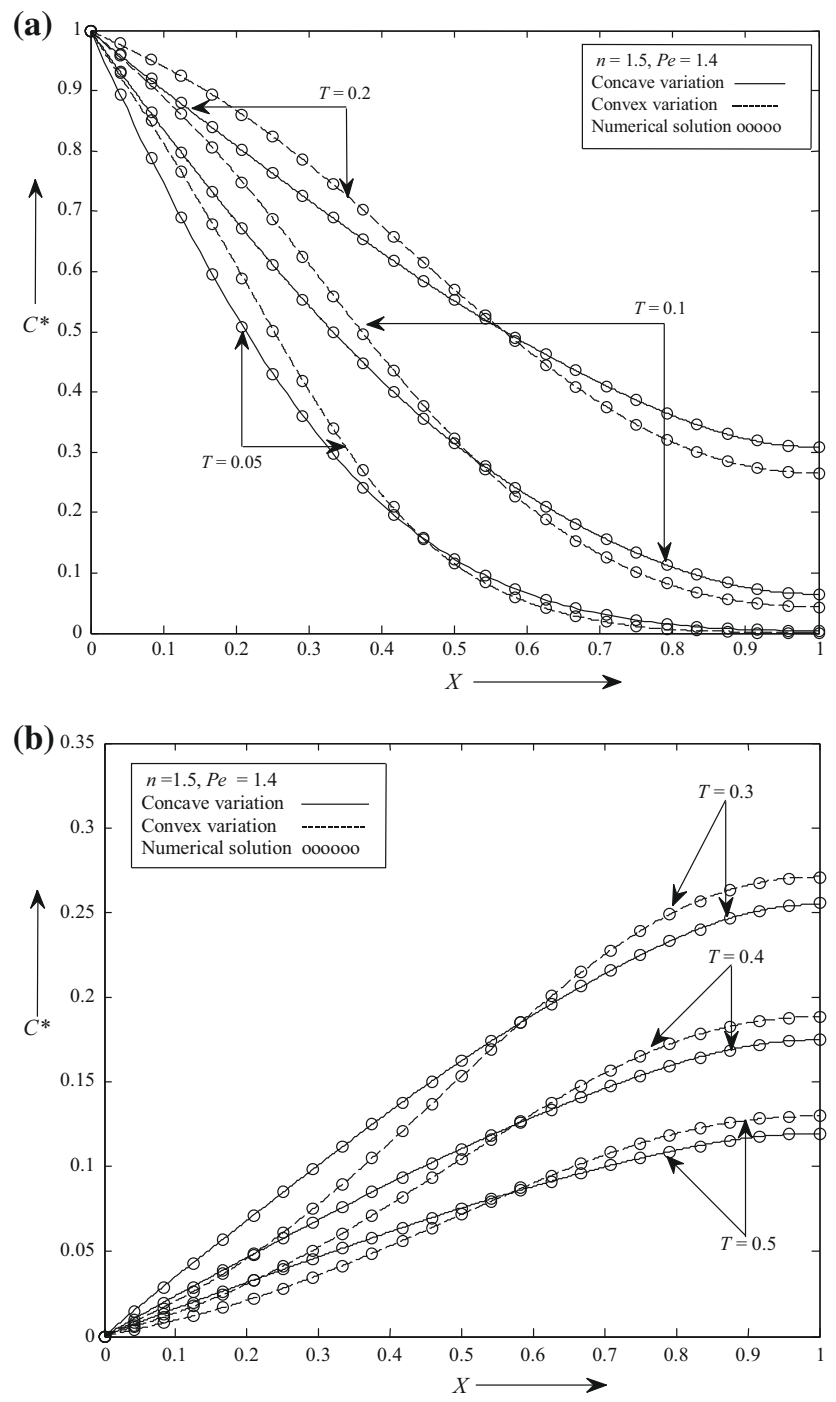

Fig. 1. Advection-dominated solute transport pattern $(\mathrm{Pe}=1.4, \ell=1.0 \mathrm{~km})$ in the fractal framework using the index value $n=1.5$ in equation (3): (a) in the presence of the uniform source, evaluated from the combination of equations (34a) and (37a) for concave spatial dispersivity and from equations (34a) and (40a) for convex variation, and (b) in the absence of source, evaluated from the combination of equations (34b) and (37b) for concave spatial dispersivity and from equations (34b) and (40b) for convex variation. The curves marked with circles depict the numerical solution of the respective problem obtained using MATLAB solver pdepe. 

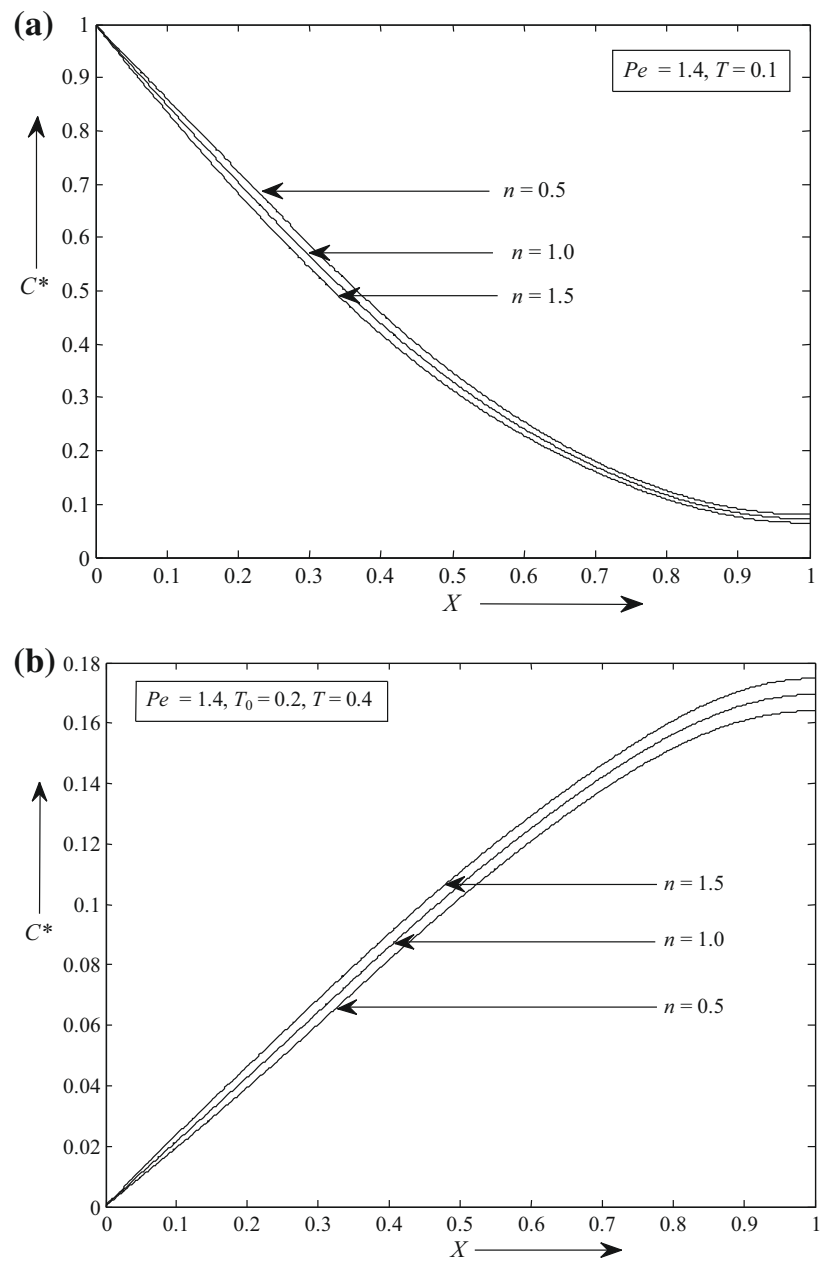

Fig. 2. Comparison of advection dominated solute transport pattern $(\mathrm{Pe}=1.4, \ell=1.0 \mathrm{~km})$ with concave spatial dispersivity using the index values $n=0.5,1.0$ and 1.5 in equation (3): (a) in the presence of uniform source, evaluated from equation (34a), in which coefficients are given in equations (35a), (36a) and (37a), respectively, and (b) in the absence of the source, evaluated from equation (34b), in which coefficients are given in equations (35b), (36b) and (37b), respectively.

to the $n$th power of velocity, with $1 \leq n \leq 2$, where also the closed-form analytical solutions were obtained for three values 1.0, 1.5 and 2.0 of the index $n$, with the first five terms of the Fourier series. Each of the proposed solutions is found matching with the numerical solution of the respective solute transport problem, obtained by using MATLAB solver pdepe, with very less percentage of approximation error. It is shown that the convergence of the analytical solutions is stable in case the Peclet number varies from a value less than 1 to a value greater than 1 in the same domain. Also, there is absolutely no change in the convergence with five terms in case the domain is lengthened to double of its length.
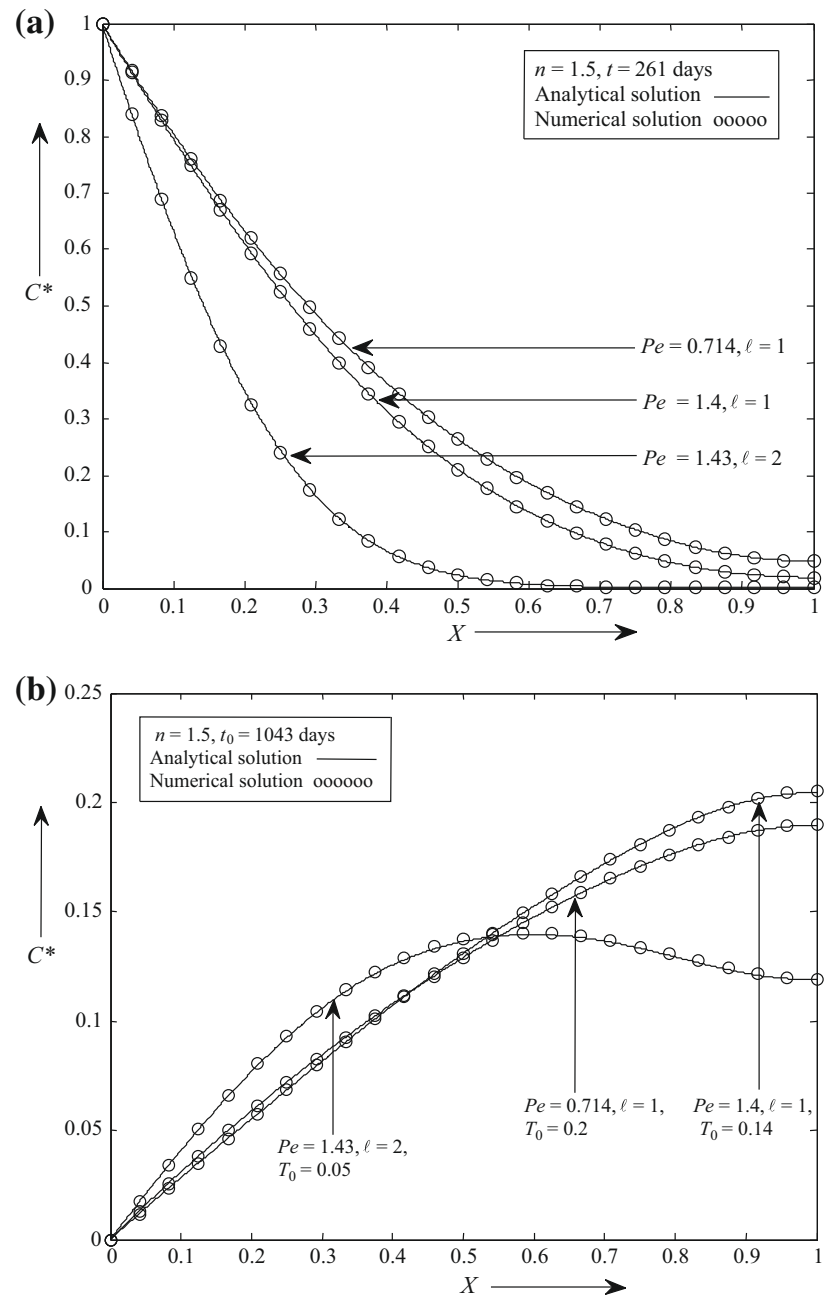

Fig. 3. Comparison of solute transport pattern when (i) advection is dominant $(\mathrm{Pe}=1.4, \ell=1.0 \mathrm{~km})$ (ii) dispersion is dominant $(\mathrm{Pe}=0.714, \ell=1.0 \mathrm{~km}$ ) and (iii) dispersion is dominant in a longer domain $(\mathrm{Pe}=1.43, \ell=2.0 \mathrm{~km})$, with concave spatial dispersivity using the index value $n=1.5$ in equation (3): (a) in the presence of uniform source, evaluated from equation (34a), in which coefficients are given in equations (37a), (38a) and (39a), respectively, and (b) in the absence of the source evaluated from equation (34b), in which coefficients are given in equations (37b), (38b) and (39b), respectively. The curves marked with circles depict the numerical solution of the respective problem obtained using MATLAB solver pdepe.

\section{Solving the governing equations using EFSM}

A one-dimensional ADE may be written in the general form as (Scheidegger 1954; Bear and Bachmat 1967; Metheron and de Marsily 1980)

$$
\frac{\partial c}{\partial t}=\frac{\partial}{\partial x}\left[D(x, t) \frac{\partial c}{\partial x}-u(x, t) c(x, t)\right], \quad 0 \leq x \leq \ell,
$$


where $D(x, t)$ is the dispersion coefficient of the dispersing mass through a heterogeneous porous medium, and $u(x, t)$ is the velocity of the groundwater flow and $\ell$ is length of the domain. Groundwater velocity is considered uniform. Dispersion coefficient is supposed to be proportional to the $n$th power of a nonhomogeneous quadratic function where the range of index $n$ is discussed in the final paragraph of the preceding section. So we consider

$$
u=u_{0}
$$

and

$$
D=D_{0}\left(b_{0}+a_{1} x+a_{2} x^{2}\right)^{n},
$$

where $u_{0}$ is uniform velocity, $D_{0}$ is the uniform dispersion coefficient, the index $n$ is considered to be $0.5,1.0$ and $1.5, b_{0}$ is a constant and coefficients $a_{1}$ and $a_{2}$ are the heterogeneity parameters such that $a_{1} x$ and $a_{2} x^{2}$ are non-dimensional terms. For an integer value $n=1$, the spatial dependence of the dispersivity will be quadratic in the Euclidean sense, whereas for any real value like $n=0.5$ and 1.5, the spatial dependence will be within the fractal framework. In both the frameworks, variation is considered to be of concave or convex (see sections 3.1 and 3.2). Thus ADE in equation (1) may be written as

$$
\begin{aligned}
\frac{\partial c}{\partial t}= & \frac{\partial}{\partial x}\left[D_{0}\left(b_{0}+a_{1} x+a_{2} x^{2}\right) \frac{n}{\partial x}-u_{0} c(x, t)\right], \\
& 0 \leq x \leq \ell .
\end{aligned}
$$

The analytical solutions of equation (4) are obtained for $n=0.5,1.0$ and 1.5, respectively.

To get the solution, one condition with respect to time variable and two conditions with respect to position variable are chosen. In the ADE, the derivative with respect to the time variable is of the first order, so it is possible to assume only the solution type or the first type condition (Dirichlet condition) in the time variable. It is usually referred to as an initial condition. The two conditions in the position variable are defined at two different positions, hence are referred to as boundary conditions. In the present context, initial and boundary conditions are assumed as follows:

An initially solute-free domain is considered before the pollutant is introduced. It is represented by a homogeneous initial condition as

$$
c(x, t=0)=0, \quad 0 \leq x \leq \ell .
$$

A uniform continuous point source of pollutant is assumed to be introduced at the origin of the finite domain. It is assumed to occur until a certain time period $t=t_{0}$, beyond which it is assumed not to exist. It is defined by a solution type (first type) pulse condition. In the presence of the source, this condition is non-homogeneous as

$$
c(x=0, t)=c_{0}, \quad 0<t \leq t_{0},
$$

where $c_{0}$ is a reference concentration, and in the absence of the source, it is a homogeneous condition as

$$
c(x=0, t)=0, \quad t>t_{0} .
$$

A flux (second type) or mixed type (third type) condition is a more appropriate condition than the solution type condition at the other end of a finite domain (You and Zhan 2013). A homogeneous flux type condition is assumed as

$$
\frac{\partial c}{\partial x}(x=\ell)=0, \quad t \geq 0 .
$$

It is convenient to proceed with non-dimensional variables, which are introduced as follows:

$$
\begin{aligned}
& C^{*}=c / c_{0}, \quad X=x / \ell, \quad T=D_{0} t / \ell^{2}, \\
& T_{0}=D_{0} t_{0} / \ell^{2}, \quad \mathrm{Pe}=u_{0} \ell / D_{0}, \\
& b_{1}=a_{1} \ell, \quad b_{2}=a_{2} \ell^{2},
\end{aligned}
$$

where Pe is referred to as the Peclet number. The $\mathrm{ADE}$ in equation (4) becomes

$$
\begin{aligned}
& \frac{\partial C^{*}}{\partial T}=\left(b_{0}+b_{1} X+b_{2} X^{2}\right) \frac{{ }_{n} \partial^{2} C^{*}}{\partial X^{2}} \\
& -\left[\mathrm{Pe}-n\left(b_{1}+2 b_{2} X\right)\left(b_{0}+b_{1} X+b_{2} X^{2}\right)^{n-1}\right] \frac{\partial C^{*}}{\partial X}
\end{aligned}
$$

or

$$
\frac{\partial C^{*}}{\partial T}=f_{1}(X) \frac{\partial^{2} C^{*}}{\partial X^{2}}-f_{2}(X) \frac{\partial C^{*}}{\partial X}
$$

where

$$
\begin{gathered}
f(X)=b_{0}+b_{1} X+b_{2} X^{2}, \\
f_{1}(X)=[f(X)]^{n}=f^{n}(X)
\end{gathered}
$$

and

$$
\begin{aligned}
f_{2}(X) & =\mathrm{Pe}-\frac{\mathrm{d}}{\mathrm{d} X} f_{1}^{n}(X) \\
& =\mathrm{Pe}-n\left(b_{1}+2 b_{2} X\right)\left(b_{0}+b_{1} X+b_{2} X^{2}\right)^{n-1} .
\end{aligned}
$$


The initial condition in equation (5) becomes

$$
C^{*}(X, T=0)=0, \quad 0 \leq X \leq 1,
$$

the boundary condition in equation (6a) in the presence of the source becomes

$$
C^{*}(X=0, T)=1, \quad 0<T \leq T_{0}
$$

and that in equation (6b) in the absence of source becomes

$$
C^{*}(X=0, T)=0, \quad T>T_{0} .
$$

The second boundary condition in equation (7) becomes

$$
\frac{\partial C^{*}}{\partial X}(X=1)=0, \quad T>0
$$

\subsection{In the presence of the source}

To solve the system of equations (9-11a) and (12) using EFSM, an SLP has to be chosen as an auxiliary system. An SLP is a homogeneous eigenvalue boundary value problem, the associated ODE has a self-adjoint operator. In its conformity, the boundary condition in equation (11a) is also made homogeneous through a transformation:

$$
C^{*}(X, T)=C(X, T)+1,
$$

the $\mathrm{ADE}$ and the conditions become

$$
\begin{gathered}
\frac{\partial C}{\partial T}=f_{1}(X) \frac{\partial^{2} C}{\partial X^{2}}-f_{2}(X) \frac{\partial C}{\partial X}, \\
C(X, T=0)=-1, \quad 0 \leq X \leq 1, \\
C(X=0, T)=0, \quad 0<T \leq T_{0}, \\
\frac{\partial C}{\partial X}(X=1)=0, \quad T>0 .
\end{gathered}
$$

The ODE of the SLP with the advective term and in terms of eigenvalue, $\lambda$ is chosen as

$$
\frac{\mathrm{d}^{2} \psi}{\mathrm{d} X^{2}}+\frac{\mathrm{d} \psi}{\mathrm{d} X}+(1+\lambda) \psi=0
$$

Equation (18) may be put in the explicit form with a self-adjoint operator as

$$
\frac{\mathrm{d}}{\mathrm{d} X}\left[\mathrm{e}^{X} \frac{\mathrm{d} \psi}{\mathrm{d} X}\right]+(1+\lambda) \mathrm{e}^{X} \psi=0 .
$$

This form shows that the weight function of the $\mathrm{SLP}$ is $\mathrm{e}^{X}$. The two boundary conditions associated with the SLP are considered to be of the same first type as associated with the ADE in equations (16 and 17), which are

$$
\psi=0 \quad \text { at } X=0
$$

and

$$
\frac{\mathrm{d} \psi}{\mathrm{d} X}=0 \quad \text { at } X=1 .
$$

The trivial solution of the problem given by equations $(18-20)$ is $\psi=0$. The non-trivial solutions are called the eigenfunctions belonging to each eigenvalue $\lambda_{m}$, and may be obtained as (Haberman 1987; Guenther and Lee 1988; Kreyszig 2014)

$$
\psi_{m}(X)=\exp (-X / 2) \sin \left(\alpha_{m} X\right)
$$

where $\alpha_{m}=1 / 2 \sqrt{3+4 \lambda_{m}}, m=1,2,3, \ldots$, are the roots of $2 \alpha_{m} \cot \alpha_{m}=0$. The functions in equation (21) are orthogonal to each other with respect to the weight function $\exp (X)$. For convenience, an orthogonal function $\psi_{m}(X)$ is replaced by its orthonormal function. It is defined as

$$
\phi_{m}(X)=\frac{\psi_{m}(X)}{\left\|\psi_{m}(X)\right\|},
$$

where $\left\|\psi_{m}(X)\right\|^{2}=\int_{0}^{1} \mathrm{e}^{X} \psi_{m}^{2}(X) \mathrm{d} X$ is the square of the norm of $\psi_{m}(X)$ with reference to a weight function $\mathrm{e}^{X}$. So the orthonormal function is

$$
\phi_{m}=\frac{1}{\left\|\psi_{m}(X)\right\|} \exp (-X / 2) \sin \left(\alpha_{m} X\right) .
$$

As $\phi_{m}(X)$ is the solution of equation (18) and satisfies the two conditions in equations (19) and (20), using $\dot{\phi}=\mathrm{d} \phi / \mathrm{d} X$, etc., we have

$$
\begin{aligned}
\ddot{\phi}_{m}= & -\dot{\phi}_{m}-\left(1+\lambda_{m}\right) \phi_{m} \\
= & -\frac{1}{2}\left(1+2 \lambda_{m}\right) \phi_{m} \\
& -\frac{1}{\left\|\psi_{m}(X)\right\|} \exp (-X / 2) \alpha_{m} \cos \left(\alpha_{m} X\right) .
\end{aligned}
$$

The orthonormal functions are also orthogonal to each other with reference to the weight function $\mathrm{e}^{X}$, i.e.,

$$
\int_{0}^{1} \mathrm{e}^{X} \phi_{m}(X) \phi_{p}(X) \mathrm{d} X=\left\{\begin{array}{cc}
1, & m=p \\
0, & m \neq p .
\end{array}\right.
$$

Therefore, the solution of the dispersion problem defined by equations (14-17) may be assumed in the form of the EFS as 


$$
C(X, T)=\sum_{m=1}^{N \rightarrow \infty} \beta_{m}(T) \phi_{m}(X),
$$

where $N$ is a finite number and $\beta_{m}(T)$ are the timedependent coefficients. This solution is substituted in equations (14 and 15). Equations (23 and 24) are used in the resulting equations, then both the equations are multiplied by $\mathrm{e}^{X} \phi_{p}(X)$ and integrated over the domain $0 \leq X \leq 1$. From equation (14) we may have

$$
\begin{aligned}
& \sum_{m=1}^{N} \frac{\mathrm{d} \beta_{m}(T)}{\mathrm{d} T} \int_{0}^{1} \mathrm{e}^{X} \phi_{m} \phi_{p} \mathrm{~d} X+\frac{1}{2} \sum_{1}^{N} \beta_{m}(T) \\
& \quad \times \int_{0}^{1}\left\{\left(1+2 \lambda_{m}\right) f_{1}(X)-f_{2}(X)\right\} \mathrm{e}^{X} \phi_{m} \phi_{p} \mathrm{~d} X \\
& \quad+\sum_{1}^{N} \beta_{m}(T) \frac{1}{\left\|\psi_{m}(X)\right\|} \\
& \quad \times \int_{0}^{1}\left\{f_{1}(X)+f_{2}(X)\right\} \alpha_{m} \cos \left(\alpha_{m} X\right) \sin \left(\alpha_{p} X\right) \mathrm{d} X=0 .
\end{aligned}
$$

The first suffix $m$ is varied from 1 to $N$, then for $p=1,2, \ldots, N$, the above equation becomes a system of $N$ first-order homogeneous ODEs, which may be put in the matrix form as

$$
\boldsymbol{Q} \frac{\mathrm{d}}{\mathrm{d} T} \boldsymbol{\beta}_{m}(\boldsymbol{T})+\boldsymbol{R} \boldsymbol{\beta}_{m}(\boldsymbol{T})=0
$$

where $\boldsymbol{Q}=\left\{a_{m, p}\right\}$ and $\boldsymbol{R}=\left\{b_{m, p}+c_{m, p}\right\}$ are the square matrices of the order $N \times N$, and

$$
a_{m, p}=\int_{0}^{1} \mathrm{e}^{X} \phi_{m} \phi_{p} \mathrm{~d} X .
$$

Using the orthogonal property given in equation $(25)$, it is evident that all the diagonal elements $(p=m)$ of matrix $\boldsymbol{Q}$ are one and non-diagonal elements $(p \neq m)$ are zero, hence matrix $\boldsymbol{Q}$ is nothing but a unit matrix $\boldsymbol{I}$. Using the expressions for orthonormal functions from equation (24), and the expression $\lambda_{m}=\alpha_{m}^{2}-3 / 4$, occurring in equation (21), the elements of matrices $\boldsymbol{R}$ may be calculated from the two integrals as follows:

$$
\begin{aligned}
b_{m, p}= & \frac{1}{2\left\|\psi_{m}(X)\right\|\left\|\psi_{p}(X)\right\|} \int_{0}^{1}\left\{\left(1+2 \lambda_{m}\right) f_{1}(X)\right. \\
& \left.-f_{2}(X)\right\} \sin \left(\alpha_{m} X\right) \sin \left(\alpha_{p} X\right) \mathrm{d} X
\end{aligned}
$$

and

$$
\begin{aligned}
c_{m, p} & =\frac{1}{\left\|\psi_{m}(X)\right\|\left\|\psi_{p}(X)\right\|} \\
& \times \int_{0}^{1}\left\{f_{1}(X)+f_{2}(X)\right\} \alpha_{m} \cos \left(\alpha_{m} X\right) \sin \left(\alpha_{p} X\right) \mathrm{d} X,
\end{aligned}
$$

where the expressions for $f_{1}(X)$ and $f_{2}(X)$ may be written from equations $(9 \mathrm{a}-\mathrm{c})$. Also from equation (15) we will get the system of initial conditions as

$$
\begin{aligned}
\boldsymbol{\beta}_{\boldsymbol{m}}(\mathbf{0}) & =-\int_{0}^{1} \mathrm{e}^{X} \phi_{m}(X) \mathrm{d} X \\
& =-\frac{1}{\left\|\psi_{m}(X)\right\|} \int_{0}^{1} \mathrm{e}^{X / 2} \sin \left(\alpha_{m} X\right) \mathrm{d} X .
\end{aligned}
$$

The system of ODE in equation (28) and the system of the initial condition (29) may be conveniently solved using the Laplace integral transform technique. To that end, the matrix $\boldsymbol{R}$ is written in the decomposed form as $\boldsymbol{R}=\boldsymbol{R}_{\mathbf{1}} \boldsymbol{D} \boldsymbol{R}_{\mathbf{1}}^{-\mathbf{1}}$, where $\boldsymbol{D}$ is the diagonal matrix formed by the eigenvalues of matrix $\boldsymbol{R}$, and the eigenvectors corresponding to each eigenvalue form matrix $\boldsymbol{R}_{\mathbf{1}}$. This method is valid when the eigenvalues of matrix $\boldsymbol{R}$ are different and non-zero. The coefficient matrix $\boldsymbol{R}$ in equation (28) is replaced by its decomposed form given above. Then, taking the Laplace integral transform of equation (28) and using the initial condition in equation (29) in that process, we may have

$$
\overline{\boldsymbol{\beta}}_{\boldsymbol{m}}(s)=\boldsymbol{R}_{\mathbf{1}}(s \boldsymbol{I}+\boldsymbol{D})^{-\mathbf{1}} \boldsymbol{R}_{\mathbf{1}}^{-\mathbf{1}} \boldsymbol{\beta}_{\boldsymbol{m}}(\mathbf{0}),
$$

where

$$
\overline{\boldsymbol{\beta}}_{\boldsymbol{m}}(s)=\int_{0}^{\infty} \boldsymbol{\beta}_{\boldsymbol{m}}(T) \mathrm{e}^{-s T} \mathrm{~d} T
$$

or

$$
\overline{\boldsymbol{\beta}}_{\boldsymbol{m}}(s)=\boldsymbol{R}_{\mathbf{1}}\left[\frac{\boldsymbol{I}}{s \boldsymbol{I}+\boldsymbol{D}}\right] \boldsymbol{R}_{1}^{-1} \boldsymbol{\beta}_{\boldsymbol{m}}(\mathbf{0}),
$$

where $s$ is the Laplace transform parameter, and the variable with an overbar on the left-hand side occurs in the Laplacian domain, the matrix $\boldsymbol{I}$ is a unit matrix and the matrix $\boldsymbol{\beta}_{\boldsymbol{m}}(\mathbf{0})$ is given in equation (29).

Let $v_{1}, v_{2}, \ldots, v_{N}$ be the eigenvalues of the matrix $\boldsymbol{R}_{\mathbf{1}}$. Taking the inverse Laplace transform of equation (30a), we get the $N$ time-dependent coefficients of equation (26) as

$$
\begin{aligned}
& {\left[\beta_{1}(T), \beta_{2}(T), \ldots, \beta_{N}(T)\right]^{\prime}} \\
& =\boldsymbol{R}_{\mathbf{1}} \operatorname{diag}\left[\mathbf{e}^{v_{1} T}, \mathbf{e}^{v_{2} T}, \ldots, \mathbf{e}^{v_{N} T}\right] \boldsymbol{R}_{\mathbf{1}}^{-1} \boldsymbol{\beta}_{\boldsymbol{m}}(\mathbf{0}),
\end{aligned}
$$


where the left-hand side is a column matrix of order $N \times 1$ written as a transpose of a row matrix of order $1 \times N$. Using these coefficients in equation (26), the solution for concentration in non-dimensional variables, $C^{*}(X, T)$ may be written from equation (13).

\subsection{In the absence of the source}

Concentration at $t=t_{0}$ in the domain $0 \leq x \leq \ell$ will serve the initial concentration in the absence of the source that is in the time domain $t>t_{0}$. It will get attenuated with time, subject to the zero concentration at the origin $(x=0)$ and no concentration flux across the boundary $(x=\ell)$, given by equations ( $6 \mathrm{~b}$ and 7 ), respectively. In the absence of the source, both the boundary conditions are homogeneous and so the transformation in equation (13) is not required. As a result, the concentration attenuation pattern may be obtained from the solution of the $\mathrm{ADE}$ in equations $(9,11 \mathrm{~b}$ and 12$)$, subject to the initial concentration $C^{*}\left(X, T=T_{0}\right)$ obtained in the previous section. Thus, in the absence of the source, the desired solution in non-dimensional variables will be

$$
C^{*}(X, T)=\sum_{m=1}^{N \rightarrow \infty} \beta_{m}(T) \phi_{m}(X),
$$

where the time-dependent coefficients will have the expression

$$
\begin{aligned}
& {\left[\beta_{1}(T), \beta_{2}(T), \ldots, \beta_{N}(T)\right]^{\prime}} \\
& \quad=\boldsymbol{R}_{\mathbf{1}} \operatorname{diag}\left[\mathrm{e}^{v_{1} T}, \mathrm{e}^{v_{2} T}, \ldots, \mathrm{e}^{v_{N} T}\right] \boldsymbol{R}_{\mathbf{1}}^{-1} C^{*}\left(X, T=T_{0}\right) .
\end{aligned}
$$

\section{Analytical solutions}

Analytical solutions are obtained for both concavetype and convex-type dispersivities in sections 3.1 and 3.2, respectively. In section 3.1, solutions are obtained for five cases, depending upon three values of index $n$ in equation (3) and two Peclet numbers. In section 3.2, only one solution is obtained for one value of the index. Each solution is obtained in terms of only the first five terms $(N=5)$ of the EFS in equation (26). The solutions are also obtained with larger number of terms of the Fourier series in the case of concave dispersivity for a real value $n=1.5$ but only insignificant variation in the solutions is obtained because the values of time-dependent coefficients $\beta_{m}(T)$, for $m>5$, become almost zero. The same feature has been observed in the case of the concave quadratic function and the index values are $n=0.5$ and 1.0. Table 1 shows this finding. It contains the concentration values in the presence of the source, for $N=5,7$ and 8 terms of the EFS in the case of concave quadratic expression and the value of the index is $n=1.5$. The solutions in the presence of source, with seven and eight terms of the Fourier series along with the time-dependent coefficients in each of the two solutions are provided in the

\begin{tabular}{|c|c|c|c|c|c|c|c|c|}
\hline \multirow[b]{2}{*}{$X$} & \multicolumn{4}{|c|}{$T=0.02$} & \multicolumn{4}{|c|}{$T=0.1$} \\
\hline & $N=5$ & $N=7$ & $N=8$ & Num. Sol. & $N=5$ & $N=7$ & $N=8$ & Num. Sol. \\
\hline 0 & 1.0000 & 1.0000 & 1.0000 & 1.0000 & 1.0000 & 1.0000 & 1.0000 & 1.0000 \\
\hline 0.1 & 0.5900 & 0.5868 & 0.5859 & 0.5856 & 0.8358 & 0.8373 & 0.8374 & 0.8384 \\
\hline 0.2 & 0.2899 & 0.2849 & 0.2834 & 0.2916 & 0.6837 & 0.6834 & 0.68277 & 0.6849 \\
\hline 0.3 & 0.1157 & 0.1132 & 0.1130 & 0.1264 & 0.5435 & 0.5420 & 0.5421 & 0.5453 \\
\hline 0.4 & 0.0372 & 0.0377 & 0.0377 & 0.0484 & 0.4191 & 0.4189 & 0.4188 & 0.4230 \\
\hline 0.5 & 0.0100 & 0.0102 & 0.0097 & 0.0164 & 0.3143 & 0.3143 & 0.3137 & 0.3191 \\
\hline 0.6 & 0.0003 & 0.0020 & 0.0021 & 0.0049 & 0.2292 & 0.2278 & 0.2277 & 0.2336 \\
\hline 0.7 & 0.0007 & 0.0007 & 0.0005 & 0.0013 & 0.1612 & 0.1601 & 0.1597 & 0.1660 \\
\hline 0.8 & 0.0006 & 0.0003 & 0.0002 & $2.9946 \times 10^{-4}$ & 0.1093 & 0.1094 & 0.1091 & 0.1155 \\
\hline 0.9 & 0.0011 & 0.0004 & 0.0004 & $5.9437 \times 10^{-5}$ & 0.0760 & 0.0756 & 0.0755 & 0.0823 \\
\hline 1.0 & 0.0001 & 0.0007 & 0.0005 & $1.8019 \times 10^{-5}$ & 0.0645 & 0.0635 & 0.0630 & 0.0695 \\
\hline
\end{tabular}

Table 1. Concentration attenuation of a uniform continuous source for spatial dispersivity of concave type for $n=1.5$, using the solutions equation (26) with five, seven and eight terms, respectively.

In the presence of source, the solution with five terms is given by equations (34a) and (37a), and those for seven and eight terms are provided in the Appendix. The analytical solutions are compared with the respective numerical solution (numerical values in the fourth column at each time). 
Appendix. It establishes the convergence of all solutions obtained in this paper for $N=5$.

Thus, using expressions of orthonormal functions from equation (23) in the EFS in equation (26), the analytical solution in the presence of the source may be written from equation (13) as

$$
\begin{aligned}
& C^{*}(X, T)=1+\exp (-X / 2) \\
& \quad \times \sum_{m=1}^{5} \beta_{m}(T) \frac{1}{\left\|\psi_{m}(X)\right\|} \sin \left(\alpha_{m} X\right)
\end{aligned}
$$

and that in the absence of the source may be written from equation (32) as

$$
\begin{aligned}
& C^{*}(X, T)=\exp (-X / 2) \\
& \quad \times \sum_{m=1}^{5} \beta_{m}(T) \frac{1}{\left\|\psi_{m}(X)\right\|} \sin \left(\alpha_{m} X\right),
\end{aligned}
$$

where $\alpha_{m}$ are the roots of the transcendental equation associated with equation (21), and the five time-dependent coefficients $\beta_{m}(T)$ of the solution in equation (34a) will be obtained from equation (31) and those of the solution in equation (34b) will be obtained from equation (33). Now these coefficients are obtained in different cases discussed below.

\subsection{Concave dispersivity}

In equation (3), the coefficients are considered as $b_{0}=0.75, a_{1}=1$ and $a_{2}=-1$. Using the non- dimensional variables in equation (8), we may write the velocity and dispersion coefficients as $u=u_{0}$ and $D=D_{0}\left(0.75+X-X^{2}\right)^{n} ; \quad 0 \leq X \leq 1$, where $n=0.5,1.0$ and 1.5 are considered. The quadratic expression $f(X)=0.75+X-X^{2}$ takes on the value of 0.75 at the end, $X=0$ and $X=1$, and a higher value 1.0 in the middle, $X=0.5$. It shows that the spatial variation of the function $f(X)$ hence that of the dispersivity (ratio of the dispersion coefficient and velocity) is also of the concave type. The solutions are obtained for the index values $n=0.5,1.0$ and 1.5 in the first three sub-cases, respectively. It means the solutions for the first and third values of the index, which are fractional values, are obtained in the fractal framework while that for the second value which is an integer, is obtained in the Euclidean frame work. In each of the three subcases, the Peclet number $\mathrm{Pe}=\left(u_{0} \ell / D_{0}\right)$ is greater than 1.0, which means the solute transport is dominated by advection. In the fourth sub-case, the Peclet number is less than one representing the dispersion-dominated solute transport. The solution in this case is obtained for $n=1.5$. In the fifth case, the solution is obtained for dispersion-dominated solute transport but in a domain of double length than that in the previous cases for $n=1.5$.

\subsubsection{Index $n=0.5$ and advection-dominated solute transport}

The values to the input variables are considered to be: length of domain $\ell=1.0 \mathrm{~km}$, uniform dispersion coefficient $D_{0}=0.10 \mathrm{~km}^{2} / \mathrm{yr}$ and uniform velocity $u_{0}=0.14 \mathrm{~km} / \mathrm{yr}$. So the Peclet number is $\mathrm{Pe}=$ $\left(u_{0} \ell / D_{0}\right)=1.4$. This value represents the advection-dominated solute transport. As the number of terms in equations (31) and (33) is five, the elements of matrix $\boldsymbol{R}$ of order $5 \times 5$ are calculated using the integrals in equations (28b) and (28c). Thereafter, the eigenvalues of the coefficient matrix $\boldsymbol{R}$ are evaluated to be: $v_{1}=186.5790, v_{2}=4.0520, v_{3}=23.1201, v_{4}=117.6983$ and $v_{5}=60.6049$. Next, the matrix of eigenvectors corresponding to the five eigenvalues is evaluated as

$$
\boldsymbol{R}_{\mathbf{1}}=\left(\begin{array}{rrrrr}
-0.0352 & -0.9949 & 0.3821 & -0.0698 & -0.1262 \\
0.0464 & 0.1009 & -0.9104 & 0.0657 & 0.2956 \\
-0.0356 & -0.0028 & 0.1566 & -0.2627 & -0.9288 \\
0.2861 & 0.0051 & -0.0183 & 0.9362 & 0.1808 \\
-0.9558 & -0.0001 & 0.0133 & -0.2131 & -0.0360
\end{array}\right) .
$$


Then, its inverse, $\boldsymbol{R}_{\mathbf{1}}^{\mathbf{1}}$ is obtained. Finally, using equation (29), matrix $\boldsymbol{\beta}_{\boldsymbol{m}}(\mathbf{0})$ is evaluated. Using these matrices in equation (31), the five coefficients of equation (34a) are obtained as

$$
\left(\begin{array}{l}
\beta_{1}(T) \\
\beta_{2}(T) \\
\beta_{3}(T) \\
\beta_{4}(T) \\
\beta_{5}(T)
\end{array}\right)=\left(\begin{array}{rrrrr}
-0.0055 & -1.4317 & 0.2304 & 0.0175 & -0.0452 \\
0.0072 & 0.1452 & -0.5489 & -0.0165 & 0.1058 \\
-0.0055 & -0.0040 & 0.0944 & 0.0659 & -0.3323 \\
0.0445 & 0.0073 & -0.0110 & -0.2347 & 0.0647 \\
-0.1488 & -0.0001 & 0.0080 & 0.0534 & -0.0129
\end{array}\right)\left(\begin{array}{l}
\exp \left(-v_{1} T\right) \\
\exp \left(-v_{2} T\right) \\
\exp \left(-v_{3} T\right) \\
\exp \left(-v_{4} T\right) \\
\exp \left(-v_{5} T\right)
\end{array}\right)
$$

Thus a combination of equations (34a) and (35a) represents the analytical solution for the concentration level in the domain in the presence of the continuous uniform point source.

Now using this solution at $T=T_{0}$ along with other matrices obtained as above, in equation (33), the five time-dependent coefficients of equation (34b) may be obtained from equation (33) as

$$
\left(\begin{array}{l}
\beta_{1}(T) \\
\beta_{2}(T) \\
\beta_{3}(T) \\
\beta_{4}(T) \\
\beta_{5}(T)
\end{array}\right)=\left(\begin{array}{rrrrr}
0.0055 & 0.7950 & -0.2281 & -0.0175 & 0.0452 \\
-0.0072 & -0.0806 & 0.5435 & 0.0165 & -0.1058 \\
0.0055 & 0.0022 & -0.0935 & -0.0659 & 0.3323 \\
-0.0445 & -0.0041 & 0.0109 & 0.2347 & -0.0647 \\
0.1487 & 0.0001 & -0.0079 & -0.0534 & 0.0129
\end{array}\right)\left(\begin{array}{l}
\exp \left(-v_{1} T\right) \\
\exp \left(-v_{2} T\right) \\
\exp \left(-v_{3} T\right) \\
\exp \left(-v_{4} T\right) \\
\exp \left(-v_{5} T\right)
\end{array}\right) .
$$

These coefficients are used in equation (34b) to have the analytical solution in the absence of the source.

\subsubsection{Index $n=1.0$ and advection-dominated solute transport}

The input values except the value of the index are the same as in section 3.1.1, so $\mathrm{Pe}=1.4$. The eigenvalues of matrix $\boldsymbol{R}$ are evaluated as: $v_{1}=178.3824, v_{2}=113.2199, v_{3}=3.9316, v_{4}=22.2443$ and $v_{5}=57.9122$. The matrix of eigenvectors of the corresponding eigenvalues is obtained as

$$
\boldsymbol{R}_{\mathbf{1}}=\left(\begin{array}{rrrrr}
-0.0346 & 0.0692 & 0.9956 & 0.3985 & -0.1275 \\
0.0437 & -0.0555 & -0.0934 & -0.9033 & 0.3071 \\
-0.1080 & 0.2613 & 0.0064 & 0.1550 & -0.9239 \\
0.3009 & -0.9336 & -0.0056 & -0.0301 & 0.1809 \\
-0.9519 & 0.2285 & 0.0007 & 0.0163 & -0.0565
\end{array}\right)
$$

It may be noted that the elements of matrix $\boldsymbol{\beta}_{\boldsymbol{m}}(\mathbf{0})$ are independent of the values of the input variables, so this matrix will be the same as in the preceding case as well as in the other cases dealt with below. Proceeding in a similar way as in the section 3.1.1, the five coefficients of the solution in equation (31) may be obtained as

$$
\left(\begin{array}{l}
\beta_{1}(T) \\
\beta_{2}(T) \\
\beta_{3}(T) \\
\beta_{4}(T) \\
\beta_{5}(T)
\end{array}\right)=\left(\begin{array}{rrrrr}
-0.0053 & 0.0170 & -1.4410 & 0.2400 & -0.0451 \\
0.0067 & -0.0136 & 0.1352 & -0.5441 & 0.1087 \\
-0.0027 & 0.0641 & -0.0093 & 0.0934 & -0.3271 \\
0.0459 & -0.2291 & 0.0081 & -0.0181 & 0.0640 \\
-0.1452 & 0.0561 & -0.0010 & 0.0098 & -0.0200
\end{array}\right)\left(\begin{array}{l}
\exp \left(-v_{1} T\right) \\
\exp \left(-v_{2} T\right) \\
\exp \left(-v_{3} T\right) \\
\exp \left(-v_{4} T\right) \\
\exp \left(-v_{5} T\right)
\end{array}\right)
$$

It may be used in equation (34a) to get the analytical solution in the presence of the source. This solution at $T=T_{0}$ along with other matrices obtained as above, is used in equation (33) to get the five timedependent coefficients as

$$
\left(\begin{array}{l}
\beta_{1}(T) \\
\beta_{2}(T) \\
\beta_{3}(T) \\
\beta_{4}(T) \\
\beta_{5}(T)
\end{array}\right)=\left(\begin{array}{rrrrr}
0.0053 & -0.0170 & 0.7846 & -0.2372 & 0.0451 \\
-0.0067 & 0.0136 & -0.0736 & 0.5377 & -0.1087 \\
0.0027 & -0.0641 & 0.0050 & -0.0923 & 0.3271 \\
-0.0459 & 0.2291 & 0.0044 & 0.0179 & -0.0640 \\
0.1453 & -0.0561 & 0.0006 & -0.0097 & 0.0200
\end{array}\right)\left(\begin{array}{l}
\exp \left(-v_{1} T\right) \\
\exp \left(-v_{2} T\right) \\
\exp \left(-v_{3} T\right) \\
\exp \left(-v_{4} T\right) \\
\exp \left(-v_{5} T\right)
\end{array}\right)
$$


These coefficients are used in equation (34b) to have the analytical solution in the absence of the source in this particular case.

\subsubsection{Index $n=1.5$ and advection-dominated solute transport}

The input values are the same as in section 3.1.1 with $\mathrm{Pe}=1.4$. Because of the different values of $n$, the eigenvalues of the matrix $\boldsymbol{R}$ occurring as a coefficient in equation (28) are obtained as $v_{1}=170.9106$, $v_{2}=109.1658, v_{3}=3.8117, v_{4}=21.4168$ and $v_{5}=55.3054$. The matrix of eigenvectors of the corresponding eigenvalues is obtained as

$$
\boldsymbol{R}_{\mathbf{1}}=\left(\begin{array}{rrrrr}
-0.0340 & 0.0683 & 0.9962 & 0.4147 & -0.1291 \\
0.0405 & -0.0453 & -0.0858 & -0.8956 & 0.3185 \\
-0.0009 & 0.2576 & 0.0101 & 0.1544 & -0.9183 \\
0.3147 & -0.9313 & -0.0060 & -0.0415 & 0.1813 \\
-0.9477 & 0.2443 & 0.0014 & 0.0194 & -0.0762
\end{array}\right)
$$

Proceeding in a similar way as in section 3.1.1, the five coefficients in equation (31) may be obtained as

$$
\left(\begin{array}{l}
\beta_{1}(T) \\
\beta_{2}(T) \\
\beta_{3}(T) \\
\beta_{4}(T) \\
\beta_{5}(T)
\end{array}\right)=\left(\begin{array}{rrrrr}
-0.0051 & 0.0164 & -1.4500 & 0.0194 & -0.0452 \\
0.0061 & -0.0109 & 0.1249 & -0.2495 & 0.1115 \\
-0.0001 & 0.0618 & -0.0147 & 0.0929 & -0.3215 \\
0.0471 & -0.2235 & 0.0087 & -0.0250 & 0.0635 \\
-0.1420 & 0.0587 & -0.0020 & 0.0117 & -0.0267
\end{array}\right)\left(\begin{array}{l}
\exp \left(-v_{1} T\right) \\
\exp \left(-v_{2} T\right) \\
\exp \left(-v_{3} T\right) \\
\exp \left(-v_{4} T\right) \\
\exp \left(-v_{5} T\right)
\end{array}\right)
$$

Using these coefficients in equation (34a), the analytical solution in the presence of the source in this particular case may be written. Further using this solution at $T=T_{0}$ along with other matrices obtained as above in equation (33), the five time-dependent coefficients may be obtained as

$$
\left(\begin{array}{l}
\beta_{1}(T) \\
\beta_{2}(T) \\
\beta_{3}(T) \\
\beta_{4}(T) \\
\beta_{5}(T)
\end{array}\right)=\left(\begin{array}{rrrrr}
0.0051 & -0.0164 & 0.7734 & -0.2460 & 0.0452 \\
-0.0061 & 0.0109 & -0.0666 & 0.5313 & -0.1115 \\
0.0001 & -0.0618 & 0.0078 & -0.0916 & 0.3215 \\
-0.0471 & 0.2235 & -0.0047 & 0.0246 & -0.0635 \\
0.1420 & -0.0586 & 0.0011 & -0.0115 & 0.0267
\end{array}\right)\left(\begin{array}{l}
\exp \left(-v_{1} T\right) \\
\exp \left(-v_{2} T\right) \\
\exp \left(-v_{3} T\right) \\
\exp \left(-v_{4} T\right) \\
\exp \left(-v_{5} T\right)
\end{array}\right)
$$

These coefficients are used in equation (34b) to have the analytical solution in the absence of the source.

\subsubsection{Index $n=1.5$ and dispersion-dominated solute transport}

Peclet number is assumed to be less than 1 to assess the effect of dispersion dominance on the solute transport as compared to that dominated by advection. The requisite values are chosen to be $\ell=1.0 \mathrm{~km}$, $D_{0}=0.14 \mathrm{~km}^{2} / \mathrm{yr}$ and $u_{0}=0.10 \mathrm{~km} / \mathrm{yr}$. So the Peclet number is $\mathrm{Pe}=0.714$. The eigenvalues of the coefficient matrix $\boldsymbol{R}$ are evaluated as: $v_{1}=173.5220, v_{2}=2.9049, v_{3}=20.2856, \quad v_{4}=107.5757$ and $v_{5}=54.4390$, and the matrix of the eigenvectors of the corresponding eigenvalues is obtained as

$$
\boldsymbol{R}_{\mathbf{1}}=\left(\begin{array}{rrrrr}
0.0196 & -0.9986 & -0.3141 & 0.0429 & 0.0750 \\
-0.0253 & 0.0515 & 0.9419 & -0.0109 & -0.2383 \\
-0.0281 & -0.0091 & -0.1117 & 0.1956 & 0.9566 \\
-0.2237 & 0.0032 & 0.0383 & -0.9640 & -0.1339 \\
0.9737 & -0.0012 & -0.0131 & 0.1747 & 0.0681
\end{array}\right)
$$


Proceeding in a similar way as in section 3.1.1, the five coefficients of the solution in equation (33) may be obtained as

$$
\left(\begin{array}{l}
\beta_{1}(T) \\
\beta_{2}(T) \\
\beta_{3}(T) \\
\beta_{4}(T) \\
\beta_{5}(T)
\end{array}\right)=\left(\begin{array}{rrrrr}
-0.0024 & -1.3675 & 0.1481 & 0.0080 & -0.0205 \\
0.0031 & 0.0705 & -0.4441 & -0.0019 & 0.0652 \\
0.0034 & -0.0125 & 0.0527 & 0.0364 & -0.2616 \\
0.0273 & 0.0044 & -0.0181 & -0.1793 & 0.0366 \\
-0.1187 & -0.0016 & 0.0062 & 0.0325 & -0.0186
\end{array}\right)\left(\begin{array}{l}
\exp \left(-v_{1} T\right) \\
\exp \left(-v_{2} T\right) \\
\exp \left(-v_{3} T\right) \\
\exp \left(-v_{4} T\right) \\
\exp \left(-v_{5} T\right)
\end{array}\right)
$$

which may be used in equation (34a) to get the analytical solution in the presence of the source. This solution at $T=T_{0}$ along with other matrices obtained as above is used in equation (33) to get the five time-dependent coefficients as

$$
\left(\begin{array}{l}
\beta_{1}(T) \\
\beta_{2}(T) \\
\beta_{3}(T) \\
\beta_{4}(T) \\
\beta_{5}(T)
\end{array}\right)=\left(\begin{array}{rrrrr}
0.0024 & 0.6026 & -0.1455 & -0.0080 & 0.0205 \\
-0.0031 & -0.0311 & 0.4364 & 0.0019 & -0.0652 \\
-0.0034 & 0.0055 & 0.0518 & -0.0364 & 0.2616 \\
-0.0273 & -0.0019 & 0.0177 & 0.1793 & -0.0366 \\
0.1187 & 0.0007 & -0.0061 & -0.0325 & 0.0186
\end{array}\right)\left(\begin{array}{l}
\exp \left(-v_{1} T\right) \\
\exp \left(-v_{2} T\right) \\
\exp \left(-v_{3} T\right) \\
\exp \left(-v_{4} T\right) \\
\exp \left(-v_{5} T\right)
\end{array}\right)
$$

These coefficients are used in equation (34b) to have the analytical solution in the absence of the source in this particular case.

\subsubsection{Index $n=1.5$ and dispersion-dominated solute transport in a domain of double length}

In this case, the domain in which the solute mass is dispersing is considered to be of the length twice of that considered in the previous cases. We consider $\ell=2.0 \mathrm{~km}$. The solute transport is considered to be dispersion dominated. We consider $D_{0}=0.14 \mathrm{~km}^{2} / \mathrm{yr}$, and $u_{0}=0.10 \mathrm{~km} / \mathrm{yr}$. So the Peclet number is $\mathrm{Pe}=1.43$. The solution is obtained for $n=1.5$. The eigenvalues of the coefficient matrix $\boldsymbol{R}$ are evaluated as $v_{1}=170.7686, v_{2}=109.2512, v_{3}=3.8552, v_{4}=21.4719$ and $v_{5}=55.3457$. The matrix of the eigenvectors of the corresponding eigenvalues is

$$
\boldsymbol{R}_{\mathbf{1}}=\left(\begin{array}{rrrrr}
-0.0347 & 0.0695 & 0.9961 & 0.4187 & -0.1315 \\
0.0412 & -0.0469 & -0.0873 & -0.8934 & 0.3218 \\
-0.0023 & 0.2599 & 0.0102 & 0.1561 & -0.9164 \\
0.3187 & -0.9297 & -0.0061 & -0.0417 & 0.1831 \\
-0.9463 & 0.2473 & 0.0014 & 0.0197 & -0.0766
\end{array}\right)
$$

Proceeding in a similar way as in section 3.1.1, the five coefficients of the solution in equation (33) may be obtained as

$$
\left(\begin{array}{l}
\beta_{1}(T) \\
\beta_{2}(T) \\
\beta_{3}(T) \\
\beta_{4}(T) \\
\beta_{5}(T)
\end{array}\right)=\left(\begin{array}{rrrrr}
-0.0053 & 0.0169 & -0.4541 & 0.2547 & -0.0466 \\
0.0062 & -0.0114 & 0.1274 & -0.5434 & 0.1139 \\
-0.0003 & 0.0631 & -0.0149 & 0.0949 & -0.3244 \\
0.0482 & -0.2258 & 0.0089 & -0.0254 & 0.0648 \\
-0.1432 & 0.0601 & -0.0020 & 0.0120 & -0.0271
\end{array}\right)\left(\begin{array}{l}
\exp \left(-v_{1} T\right) \\
\exp \left(-v_{2} T\right) \\
\exp \left(-v_{3} T\right) \\
\exp \left(-v_{4} T\right) \\
\exp \left(-v_{5} T\right)
\end{array}\right)
$$

These coefficients are used in equation (34a) to get the analytical solution in the presence of the source. This solution at $T=T_{0}$ along with other matrices obtained as above is used in equation (33) to get the five time-dependent coefficients as

$$
\left(\begin{array}{l}
\beta_{1}(T) \\
\beta_{2}(T) \\
\beta_{3}(T) \\
\beta_{4}(T) \\
\beta_{5}(T)
\end{array}\right)=\left(\begin{array}{rrrrr}
0.0053 & -0.0168 & 0.2549 & -0.1676 & 0.0436 \\
-0.0062 & 0.0113 & -0.0223 & 0.3576 & -0.1068 \\
0.0003 & -0.0628 & 0.0026 & -0.0625 & 0.3041 \\
-0.0482 & 0.2248 & -0.0016 & 0.0167 & -0.0608 \\
0.1432 & -0.0598 & 0.0004 & -0.0079 & 0.0254
\end{array}\right)\left(\begin{array}{l}
\exp \left(-v_{1} T\right) \\
\exp \left(-v_{2} T\right) \\
\exp \left(-v_{3} T\right) \\
\exp \left(-v_{4} T\right) \\
\exp \left(-v_{5} T\right)
\end{array}\right)
$$


These coefficients are used in equation (34b) to have the analytical solution in the absence of the source in this particular case.

\subsection{Convex dispersivity}

In equation (3), the coefficients are considered as $b_{0}=1.0, a_{1}=-1$ and $a_{2}=1$. Using the nondimensional variables in equation (8), we may write the velocity and dispersion coefficients as $u=u_{0}$ and $D=D_{0}\left(1.0-X+X^{2}\right)^{n} ; 0 \leq X \leq 1$, where the solution is obtained only for a real value $n=1.5$. This value presents the spatial dependence of dispersivity in the fractal framework. The quadratic expression $f(X)=1.0-X+X^{2}$ takes on the value of 1.0 at the ends, that is, at $X=0$ and $X=1$, and a lower value 0.75 in the middle $(X=0.5)$. It shows that the spatial variation of the dispersivity is of convex type. Other requisite values are chosen to be $\ell=1.0 \mathrm{~km}, D_{0}=0.10 \mathrm{~km}^{2} / \mathrm{yr}$ and $u_{0}=0.14 \mathrm{~km} / \mathrm{yr}$. So the Peclet number is $\mathrm{Pe}=1.4$, as in the case of section 3.1.3. The eigenvalues of the coefficient matrix $\boldsymbol{R}$ are obtained as $v_{1}=149.6581, v_{2}=93.6308, v_{3}=3.7395$, $v_{4}=18.6396$ and $v_{5}=48.3832$. The matrix of the eigenvectors of the corresponding eigenvalues is obtained as

$$
\boldsymbol{R}_{\mathbf{1}}=\left(\begin{array}{rrrrr}
-0.0518 & 0.0940 & 0.9867 & 0.3985 & -0.1657 \\
0.0705 & -0.1363 & -0.1621 & -0.8946 & 0.3181 \\
-0.3145 & 0.3096 & -0.0068 & 0.2008 & -0.9081 \\
0.2847 & -0.9163 & -0.0014 & 0.0238 & 0.2121 \\
-0.9451 & 0.1929 & -0.0015 & 0.0001 & 0.0423
\end{array}\right)
$$

Proceeding in a similar way as in section 3.1.1, the five coefficients of the solution in equation (31) may be obtained as

$$
\left(\begin{array}{l}
\beta_{1}(T) \\
\beta_{2}(T) \\
\beta_{3}(T) \\
\beta_{4}(T) \\
\beta_{5}(T)
\end{array}\right)=\left(\begin{array}{rrrrr}
-0.0102 & 0.0310 & -1.4751 & 0.2960 & -0.0760 \\
0.0138 & -0.0449 & 0.2423 & -0.6644 & 0.1459 \\
-0.0264 & 0.1021 & 0.0102 & 0.1491 & -0.4165 \\
0.0559 & -0.3021 & 0.0021 & 0.0177 & 0.0973 \\
-0.1856 & 0.0636 & 0.0022 & 0.0001 & 0.0194
\end{array}\right)\left(\begin{array}{l}
\exp \left(-v_{1} T\right) \\
\exp \left(-v_{2} T\right) \\
\exp \left(-v_{3} T\right) \\
\exp \left(-v_{4} T\right) \\
\exp \left(-v_{5} T\right)
\end{array}\right)
$$

These coefficients are used in equation (34a) to get the analytical solution in the presence of the source. This solution at $T=T_{0}$ along with the other matrices obtained as above is used in equation (33) to get the five time-dependent coefficients as

$$
\left(\begin{array}{l}
\beta_{1}(T) \\
\beta_{2}(T) \\
\beta_{3}(T) \\
\beta_{4}(T) \\
\beta_{5}(T)
\end{array}\right)=\left(\begin{array}{rrrrr}
0.0102 & -0.0310 & -0.7769 & -0.2889 & 0.0760 \\
-0.0138 & 0.0449 & 0.1276 & 0.6485 & -0.1459 \\
0.0264 & -0.1021 & 0.0054 & -0.1456 & 0.4165 \\
-0.0559 & 0.3021 & 0.0011 & -0.0173 & -0.0973 \\
0.1856 & -0.0636 & 0.0012 & -0.0001 & -0.0194
\end{array}\right)\left(\begin{array}{l}
\exp \left(-v_{1} T\right) \\
\exp \left(-v_{2} T\right) \\
\exp \left(-v_{3} T\right) \\
\exp \left(-v_{4} T\right) \\
\exp \left(-v_{5} T\right)
\end{array}\right)
$$

These coefficients are used in equation (34b) to have the analytical solution in the absence of the source in this particular case.

\section{Results and discussion}

The analytical solutions obtained in terms of nondimensional variables in the different cases in the preceding section are illustrated in a non-dimensional domain $0 \leq X \leq 1$. All the solutions delineate the concentration attenuation pattern in the presence of a uniform continuous source introduced at the origin and after the source is removed forever, subject to zero concentration flux at the other end of the domain. All the three figures sufxed (a) exhibit that concentration at the origin is 1.0 at each time, in the presence of the source in accordance with the condition in equation (11a), and all the figures suffixed (b) show zero concentration at the origin at each time after the removal of the source, described by the condition in equation (11b). These figures also depict that the 
concentration at the other end $X=1$ increases with time, in the presence of source while decreases with time in the absence of source. Also, each concentration curve becomes flat as the boundary $X=1.0$ is approached which means the concentration gradient is zero there. This phenomenon is described by the condition in equation (12). The concentration level attenuates with position at a time. In the presence of the source at the origin, its concentration increases with time at a decreasing rate at a particular position in the domain $0<X<1$, so that after a certain time period, the pollution level reaches a stable state and the presence of the continuous source has no further effect on the domain, whereas after the removal of source, it decreases fast. Thus, the proposed analytical solutions elucidate all the important features of solute transport originating from a uniform continuous source in its presence as well as after its annihilation, in an efficacious way. Rehabilitation time of a polluted domain may be estimated through such study. Figure 1 shows the effect of spatial variation whether it is concave or convex, figure 2 shows the effect of the index value and figure 3 exhibits the effect of Peclet number on solute transport.

Figure 1(a and b) compares the solutions obtained with concave and convex spatially varying dispersivity, in the presence of source and in the absence of source, respectively. The common values with which both the figures are drawn are the index $n=1.5$ in equation (3), $u_{0}=0.14 \mathrm{~km} / \mathrm{yr}$ and $D_{0}=0.10 \mathrm{~km}^{2} / \mathrm{yr}$. A higher value of uniform velocity than that of uniform dispersion coefficient represents the advection-dominated solute transport in a domain of length $\ell=1.0 \mathrm{~km}$. So Peclet number is greater than one, $\mathrm{Pe}=\left(u_{0} \ell / D_{0}\right)=1.4$. In concave spatial variation, as explained at the outset of section 3.1, the maximum numerical value of $D=D_{0} f^{n}(X)$ for $n=1.5$ will occur in the middle of the domain $0 \leq X \leq 1$, which will be 0.10 , whereas at the two ends of the domain, it will be $0.10 \times 0.75^{1.5}=0.065$. It shows that the values of the dispersion coefficient remain less than that of uniform velocity at every position of the domain. So this set of values will represent advectiondominated solute transport for each value of the index $n=0.5,1.0$ and 2.0. Figure 1(a) is drawn at non-dimensional time values $T\left(D_{0} t / \ell^{2}\right)=0.5,1.0$ and 2.0 i.e., at $t=18,36$ and 73 days, respectively. The solid curves represent the solution given by the combination of equations (34a) and (37a), in the presence of source, obtained with the concave function $f(X)$, and the dashed curves represent the same obtained with a convex function $f(X)$, given by the combination of equations (34a and 40a). The concentration at the origin remains 1.0 at each time. For the concave spatial variation, in the first half of the domain, the dispersion coefficient increases, whereas in the convex variation, it decreases, so in the first half of the domain the solute transport is faster characterised by a lower concentration level than that in the case of the latter type variation. As the trend of the variation of dispersivity reverses in the second half of the domain to that in the first half, the solute transport pattern also follows the same trend in the second half. This type of effect is enhanced slightly with time as evident from figure 1(a). Figure 1(b) demonstrates how the domain of length $\ell=1.0 \mathrm{~km}$ which got polluted until $T=T_{0}=0.2$, i.e., until the 73rd day due to a uniform source at the origin gets rehabilitated once the source is annihilated. This figure depicts the combination of equations (34b and $37 \mathrm{~b}$ ) for concave spatial variation in dispersivity by solid curves and combination of equations (34b and 40b) for convex variation by dashed curves, at $T\left(>T_{0}\right)=0.3$ (110 days), 0.4 (146 days) and 0.5 (183 days). Figure $1(\mathrm{~b})$ shows that the concentration at the origin remains zero at each time. The concentration in the polluted domain gets attenuated with time. In the first half of the domain, it is faster with convex spatial variation than that with concave variation, whereas the trend reverses in the second half.

Figure 2(a and b) compares the solutions obtained using the index values $n=0.5,1.0$ and 1.5 , in the presence of the source and in the absence of the source, provided in sections 3.1.1, 3.1.2 and 3.1.3, respectively. Each solution is obtained with a concave spatial variation in the dispersivity using the same values of the length of the domain, uniform velocity and dispersion coefficient with which figure $1(\mathrm{a}$ and $\mathrm{b}$ ) is drawn, i.e., Peclet number is $\mathrm{Pe}=1.4$. Figure $2(\mathrm{a})$ is drawn at $T=0.1$, i.e., at $t=37$ th day. As evident from equation (3), the value of the dispersion coefficient increases with the index $n$, so solute transport is faster in the case of a higher value of the index shown by a lower concentration level. As the velocity is uniform throughout the domain, this trend remains unchanged until the other end. Once the source is removed forever as evident from figure 2(b), rehabilitation of the domain is faster for a lower value of index $n$. To show it the source is assumed to be 
annihilated at $T=T_{0}=0.2$ that is at $t_{0}=73 \mathrm{rd}$ day.

Figure 3(a and b) illustrates the solutions in the presence of the source and in the absence of the source, respectively. These solutions are obtained for (i) advection-dominated solute transport, with input values $u_{0}=0.14 \mathrm{~km} / \mathrm{yr}, D_{0}=0.10 \mathrm{~km}^{2} / \mathrm{yr}$, $\ell=1.0 \mathrm{~km}$, i.e., for $\mathrm{Pe}=1.4$; (ii) dispersion-dominated solute transport, with input values $u_{0}=0.10 \mathrm{~km} / \mathrm{yr}, \quad D_{0}=0.14 \mathrm{~km}^{2} / \mathrm{yr}, \quad \ell=1.0 \mathrm{~km}$ $(\mathrm{Pe}=0.714)$, and (iii) dispersion-dominated solute transport in a domain of length twice of that in the previous cases, with input values $u_{0}=0.10 \mathrm{~km} / \mathrm{yr}$, $D_{0}=0.14 \mathrm{~km}^{2} / \mathrm{yr}$, and length of the domain being twice, i.e., $\ell=2.0 \mathrm{~km}(\mathrm{Pe}=1.43)$. These solutions are obtained with concave spatial variation in the dispersivity with the index value $n=1.5$ that in the fractal framework. These are provided in sections 3.1.3, 3.1.4 and 3.1.5, respectively. Figure $3(\mathrm{a})$ is illustrated at $t=261$ th day after the introduction of the uniform source at the origin. It may be observed that the concentration level is lower in the case of the advection-dominated solute transport i.e., in the case of Peclet number greater than $1(\mathrm{Pe}=1.4)$ than that in the case of dispersion-dominated solute transport in the domain of the same length, i.e., in the case of Peclet number less than $1(\mathrm{Pe}=0.714)$. This figure also shows the special feature of the solute concentration level being much lower in a domain of longer length (Sauty 1980) by comparing the concentration curves in the case of dispersion-dominated solute transport for $\mathrm{Pe}=0.714$ and 1.43 . It may be noted that the non-dimensional time variable has different values corresponding to $t=260$ days for each Peclet number, and they are $T=0.1,0.071$ and 0.025 when $\mathrm{Pe}=1.4,0.714$ and 1.43 , respectively. The source at the origin is assumed to be removed forever at $t=t_{0}=520$ th day, and the rehabilitation pattern of the polluted domain in the three cases of the Peclet number is observed at $t=1042$ days, which gives the non-dimensional time values $T=0.4,0.29$ and 0.1 for $\mathrm{Pe}=1.4$, 0.714 and 1.43 , respectively. Similarly the non-dimensional time of elimination of the source may be evaluated as $T_{0}=0.2,0.1$ and 0.05 , for the three Peclet numbers, respectively. The rehabilitation pattern is shown in figure 3(b). In advection-dominated solute transport, rehabilitation is slower than that in the case of dispersion-dominated solute transport. In a domain of twice the length, the solute transport is much slower and the peak concentration is easily visualised near the origin.
The concentration pattern shown in figures 1(a and $\mathrm{b}$ ) and $3(\mathrm{a}$ and $\mathrm{b})$, obtained from respective analytical solutions are compared with the respective numerical solutions obtained by solving the same solute transport problem by using MATLAB solver pdepe. The concentration values obtained numerically are shown by curves marked with circles in these figures. As evident, each numerical solution is in good agreement with the respective analytical solution. The error of approximation is too less to be noticed in the figures in which analytical and numerical solutions are compared with each other. Table 1 shows the convergence criterion fully satised. As a result, sensitivity analysis (to examine how output varies with small variations in the input parameters) is not performed in this work. Basu et al. (2010) have used the graphical method by representing the sensitivity of an out variable as the root mean square deviation. Najafzadeh et al. (2018) also viewed sensitivity analysis to determine the relative importance of each input parameter for estimating water quality parameters and have compared the accuracy of the proposed methods using a few of statistical criterion, in want of explicit equations to predict it. It establishes the authenticity of the analytical solutions obtained in this paper in the form of the EFS with only the first five terms.

\section{Conclusions}

In the proposed study, analytical solutions of the one-dimensional ADE are obtained with spatial variability of dispersivity in three cases: (i) dispersivity is proportional to a non-homogeneous spatial quadratic function, (ii) square of dispersivity is proportional to the same quadratic function and (iii) square of dispersivity is proportional to the cube of this function. The first one describes the dispersivity in the Euclidean framework, whereas the last two cases are in the fractal framework. From the quadratic function both concave and convex variations are figured out. Groundwater velocity is considered uniform. EFSM is used and the analytical solutions have been obtained in the form of EFS with only the first five terms. In the previous studies which used this method but with a different name GITT (generalised integral transform technique), the convergence of the EFS was the biggest issue and it could be achieved with a large number of terms, hence the system of IVP into which the solute transport problem is reduced, had been solved numerically. Also, the number of 
terms of the EFS varied significantly from 30 to more than a 1000 terms with Peclet number, type of boundary conditions and coefficients of the ADE. Hence, analytical solutions had not been possible in the previous studies. But in the present study, the analytical solutions with only five terms of the EFS have been found to be stable and robust with respect to each of these aspects. It has been possible by begetting a new SLP with advective information. The same SLP was also used in Bharati et al. (2017, 2018) in which the dispersivity has been regarded as proportional to non-homogeneous linear spatial function in the fractal as well as Euclidean sense.

Polluting time of one freshwater porous domain due to continuously occurring point source of solute mass as well as rehabilitation time of that domain may be different from that of a different freshwater porous domain. It depends upon the heterogeneity of the aquifer, dispersing ability (represented by the dispersion coefficient) of the pollutant and the groundwater velocity. In the present study, the effects of heterogeneity, concave/convex spatial variation, dispersion/advection dominated Peclet number and of the length of the finite domain, on the solute transport are shown through three figures, and each figure has two parts, one shows the polluting pattern of a freshwater domain in the presence of the source, and the other part shows the rehabilitation pattern of the polluted domain, once the source is removed. Such analytical solutions impart reasoning to know the geological conditions of an aquifer by knowing the solute transport pattern through it.

\section{Acknowledgements}

The first and third authors acknowledge the University Grants Commission, Government of India for the financial and academic assistance in the form of a senior research fellowship.

\section{Appendix}

Here the analytical solutions are provided with seven and eight terms of the EFS in equation (26) using the concave variation of dispersivity for the index $n=1.5$ in equation (3). Peclet number is $\mathrm{Pe}=1.4$ in the domain of length $\ell=1.0 \mathrm{~km}$. Other input values are $u_{0}=0.14 \mathrm{~km} / \mathrm{yr}$ and $D_{0}=0.1 \mathrm{~km}^{2} / \mathrm{yr}$. The solution with seven terms in the presence of the source is as below:

$$
C^{*}(X, T)=1+\exp (-X / 2) \sum_{m=1}^{7} \beta_{m}(T) \frac{1}{\left\|\psi_{m}(X)\right\|} \sin \left(\alpha_{m} X\right),
$$

where $\alpha_{m}$ are the roots of the transcendental equation associated with (21), and the seven time-dependent coefficients may be obtained from equation (31) as

$$
\left(\begin{array}{l}
\beta_{1}(T) \\
\beta_{2}(T) \\
\beta_{3}(T) \\
\beta_{4}(T) \\
\beta_{5}(T) \\
\beta_{6}(T) \\
\beta_{7}(T)
\end{array}\right)=\left(\begin{array}{rrrrrrr}
-0.0015 & 0.0038 & -0.0077 & -1.4525 & 0.2523 & 0.0193 & -0.0480 \\
0.0017 & -0.0030 & 0.0088 & 0.1252 & -0.5417 & -0.0133 & 0.1152 \\
-0.0011 & 0.0041 & -0.0019 & -0.0143 & 0.0922 & 0.0720 & -0.3326 \\
0.0017 & 0.0031 & 0.0511 & 0.0089 & -0.0252 & -0.2367 & 0.0679 \\
0.0051 & 0.0309 & -0.1742 & -0.0017 & 0.0114 & 0.0547 & -0.0265 \\
0.0304 & -0.1355 & 0.0392 & 0.0019 & -0.0042 & -0.0246 & 0.0107 \\
-0.0933 & 0.0400 & -0.0235 & -0.0004 & 0.0029 & 0.0107 & -0.0053
\end{array}\right)\left(\begin{array}{l}
\exp \left(-v_{1} T\right) \\
\exp \left(-v_{2} T\right) \\
\exp \left(-v_{3} T\right) \\
\exp \left(-v_{4} T\right) \\
\exp \left(-v_{1} T\right) \\
\exp \left(-v_{6} T\right) \\
\exp \left(-v_{7} T\right)
\end{array}\right),
$$

where $v_{1}=359.9983, \quad v_{2}=267.6400, \quad v_{3}=174.8626, \quad v_{4}=3.8192, \quad v_{5}=21.4275, \quad v_{6}=107.4537, \quad v_{7}=$ 55.7066 are the seven eigenvalues of the matrix $\boldsymbol{R}$ in equation (28).

Similarly, the solution with eight terms in equation (26) may be written in the presence of the source as 


$$
C^{*}(X, T)=1+\exp (-X / 2) \sum_{m=1}^{8} \beta_{m}(T) \frac{1}{\left\|\psi_{m}(X)\right\|} \sin \left(\alpha_{m} X\right),
$$

where the eigenvalues of the matrix $\boldsymbol{R}$ in (28) are obtained as $v_{1}=481.1673, v_{2}=373.6767, v_{3}=$ $260.3778, v_{4}=176.3123, v_{5}=3.8211, v_{6}=21.4225, v_{7}=55.8164, v_{8}=107.1349$, in terms of which the time-dependent coefficients of (A3) are obtained from equation (31) as

$$
\left(\begin{array}{l}
\beta_{1}(T) \\
\beta_{2}(T) \\
\beta_{3}(T) \\
\beta_{4}(T) \\
\beta_{5}(T) \\
\beta_{6}(T) \\
\beta_{7}(T) \\
\beta_{8}(T)
\end{array}\right)=\left(\begin{array}{rrrrrrrr}
0.0010 & -0.0020 & 0.0044 & -0.0083 & -1.4534 & 0.2527 & -0.0486 & 0.0198 \\
-0.0008 & 0.0021 & -0.0035 & 0.0093 & 0.1253 & -0.5419 & 0.1158 & -0.0136 \\
0.0011 & -0.0016 & 0.0049 & -0.0024 & -0.0143 & 0.0919 & -0.3351 & 0.0738 \\
-0.0005 & 0.0023 & 0.0032 & 0.0514 & 0.0089 & -0.0253 & 0.0690 & -0.2381 \\
0.0007 & 0.0056 & 0.0393 & -0.1826 & -0.0018 & 0.0112 & -0.0263 & 0.0534 \\
0.0062 & 0.0234 & -0.1397 & 0.0450 & 0.0019 & -0.0043 & 0.0112 & -0.0257 \\
0.0259 & -0.1115 & 0.0325 & -0.0234 & -0.0003 & 0.0028 & -0.0050 & 0.0100 \\
-0.0792 & 0.0342 & -0.0220 & 0.0100 & 0.0006 & -0.0013 & 0.0031 & -0.0056
\end{array}\right)\left(\begin{array}{l}
\exp \left(-v_{1} T\right) \\
\exp \left(-v_{2} T\right) \\
\exp \left(-v_{3} T\right) \\
\exp \left(-v_{4} T\right) \\
\exp \left(-v_{5} T\right) \\
\exp \left(-v_{6} T\right) \\
\exp \left(-v_{7} T\right) \\
\exp \left(-v_{8} T\right)
\end{array}\right) .
$$

\section{References}

Adler P M 1985 Transport processes in fractals, II, stokes flow in fractal capillary networks; Int. J. Multiph. Flow $\mathbf{1 1}$ $241-254$.

Basu S K, Kumar N and Srivastava J P 2010 Modeling NPK release from spherically coated fertilizer granules; Simul. Model. Pract. Theory 18 820-835.

Bear J and Bachmat Y 1967 A generalized theory on hydrodynamic dispersion in porous media; Int. Assoc. Sci. Hydrol. Publ. 72, OSTI ID: 6035908.

Bharati V K, Singh V P, Sanskrityayn A and Kumar N 2017 Analytical solution of advection diffusion equation with spatially dependent dispersivity; J. Eng. Mech. 143(11) $1-11$.

Bharati V K, Singh V P, Sanskrityayn A and Kumar N 2018 Analytical solutions for solute transport from varying pulse source along porous media flow with spatial dispersivity in fractal \& Euclidean framework; Eur. J. Mech. B Fluids $\mathbf{7 2}$ 410-421.

Cassol M, Wortmann S and Rizza U 2009 Analytic modeling of two-dimensional transient atmospheric pollutant dispersion by double GITT and Laplace transform technique; Env. Model. Softw. 24 144-151.

Castellöes F V, Quaresma J N N and Cotta R M 2010 Convective heat transfer enhancement in low Reynolds number flows with wavy walls; Int. J. Heat Mass Transf. $532022-2034$.

Chen J-S, Ni C F and Liang C P 2008 Analytical power series solution to the two-dimensional advection-dispersion equation with distance-dependent dispersivites; Hydrol. Process. 22(24) 4670-4678.

Chen J, Chen J, Liu C, Liang C and Lin C 2011 Analytical solutions to two-dimensional advection-dispersion in cylindrical coordinates in finite domain subject to first- and thirdtype inlet boundary conditions; J. Hydrol. 405 522-531.

Cotta R M 1990 Hybrid numerical-analytical approach to nonlinear diffusion problems; Numer. Heat Transfer B Fundam. 127 217-226.
Cotta R M 1993 Integral transforms in computational heat and fluid flow; CRC Press, Boca Raton, USA.

Cotta R M, Ungs M J and Mikhailov M D 2003 Contaminant transport in finite fractured porous medium: Integral transforms and lumped-differential formulations; Ann. Nucl. Energy 30 261-285.

Cotta R M, Cotta B P, Naveira-Cotta C P and Cotta-Pereira G 2010 Hybrid integral transforms analysis of the bio-heat equation with variable properties; Int. J. Thermal Sci. 49 1510-1516.

Cussler E L 1997 Diffusion: Mass transfer in fluid systems; Cambridge University Press, Cambridge, UK, pp. 142-184.

Dale P D, Sherrat J A and Maini P K 1996 A mathematical model for collagen fiber formation during foetal and adult dermal wound healing; Proc. Roy. Soc. London B 263 653-660.

Di Federico V and Neuman S P 1998 Transport in multiscale log conductivity fields with truncated power variograms; Water Resour. Res. 34(5) 963-973.

Gao G, Zhan H, Feng S, Fu B, Ma Y and Huang G 2010 A new mobile-immobile model (MIM) for reactive solute transport with scale-dependent dispersion; Water Resour. Res. 46 W08533, https://doi.org/10.1029/2009wr00870.

Gelhar L W, Welty C and Rehfeldt K R 1992 A critical review of data on field-scale dispersion in aquifers; Water Resour. Res. 28(7) 1955-1974.

Guenther R B and Lee J W 1988 Partial differential equations of mathematics physics and integral equation; PrenticeHall, Englewood Califfs, NJ.

Guerrero J S P, Pimentel L C G, Skaggs T H and Th van Genuchten M 2009 Analytical solution of the advectiondiffusion transport equation using a change-of-variable and integral transform technique; Int. J. Heat Mass Transf. 52 $3297-3304$.

Guerrero J S P, Pimentel L C G and Skaggs T H 2013 Analytical solution for the advection- dispersion transport equation in layered media; Int. J. Heat Mass Transf. 56 274-282.

Haberman R 1987 Elementary applied partial differential equations; Prentice Hall, New Jersey. 
Hiester N K and Vermeulen T 1952 Saturation performance of ion-exchange and adsorption columns; Chem. Eng. Process. 48 505-516.

Houghton P A, Madurawe R U and Hatton T A 1988 Convective and dispersive models for dispersed phase axial mixing, the significance of poly-dispersivity effects in liquid-liquid contactors; Chem. Eng. Sci. 43 617-639.

Huang K, Th Van Genuchten M and Zhang R 1996 Exact solutions for one-dimensional transport with asymptotic scale-dependent dispersion; Appl. Math. Model. 20 298-308.

Hunt B 1998 Contaminant source solutions with scale-dependent dispersivities; J. Hydrol. Eng. 3(4) 268-275.

Hunt B 2002 Scale-dependent dispersion from a pit; J. Hydrol. $\mathbf{7}(2)$ 168-174.

Kinzelbach W P and Ackerer P 1986 Modelisation de la propogation d' un champ d' écoulement transitoire; Hydrogeologie 2 197-206.

Kumar A, Jaiswal D K and Kumar N 2009 Analytical solution of one-dimensional advection- diffusion equation with variable coefficients in a finite domain; J. Earth Syst. Sci. 118(5) 539-549.

Kreyszig E 2014 Advanced engineering mathematics; Wiley, New Delhi.

Liu C, Szecsody J E, Jonh M Z and Ball W P 2000 Use of the generalized integral transform method for solving equations of solute transport in porous media; Adv. Water Resour. 23 483-492.

Metheron G and De Marsily G 1980 Is transport in porous always diffusive? A counter example; Water Resour. Res. 16(5) 901-917.

Molz F J, Güven O and Melville J G 1983 An examination of scale-dependent dispersion coefficients; Ground Water 21 $715-725$.

Najafzadeh M, Ghaemi A and Emamgholizadeh S 2018 Prediction of water quality parameters using evolutionary computing-based formulations; Int. J. Env. Sci. Technol., https://doi.org/10.1007/s13762-018-2049-4.

Neuman S P 1990 Universal scaling of hydraulic conductivities and dispersivities in geologic media; Water Resour. Res. 26(8) 1749-1758.

Neuman S P 2016 Comment on "Is unique scaling of aquifer macrodispersivity supported by field data?" By A Zech, S Attinger, V Cvetkovic, G Dagan, P Dietrich, A Fiori, Y Rubin and G Teutsch; Water Resour. Res. 52 4199-4202.

Pang L and Hunt B 2001 Solutions and verification of a scaledependent dispersion model; J. Contam. Hydrol. 53(1-2) 21-39.

Pickens J F and Grisak G E 1981 Modeling of scale-dependent dispersion in hydrogeologic systems; Water Resour. Res. 1701-1711.

Corresponding editor: ABhiJtT MukherJeE
Qiu Y, Deng B and Kim C N 2011 Analytical solution for spatially dependent solute transport in streams with storage zone; ASCE J. Hydrol. Eng. 16 689-694.

Rehfeldt K R and Gelhar L W 1993 Stochastic analysis of dispersion in unsteady flow in heterogeneous aquifers; Water Resour. Res. 28 2085-2099.

Sauty J P 1980 An analysis of hydrodispersion transfer in aquifers; Water Resour. Res. 16 145-158.

Scheidegger A E 1954 Statistical hydrodynamics in porous media; J. Appl. Phys. 25(8) 994-1001.

Schugart R C, Friedman A, Zhao R and Sen C K 2008 Wound angiogenesis as a function of tissue oxygen tension: A mathematical model; Proc. Natl. Acad. Sci. 105 2628-2633.

Sudicky E A, Cherry J A and Frind E O 1983 Migration of contaminants in groundwater at a landfill: A case study, 4, a natural gradient dispersion test; J. Hydrol. 63 81-108.

Thorpe G R and Whitaker S 1992 Local mass and thermal equilibrium in ventilated grain bulks, part I: The development of heat and mass conservation equations; J. Stored Prod. Res. 28 15-27.

Thorpe G R, Ochoa-Tapia J A and Whitaker S 1991 The diffusion of moisture in food grains, II: Estimation of effective diffusivity; J. Stored Prod. Res. 27 11-30.

Todd A 1989 On the simulation of incompressible miscible displacement in a naturally fractured petroleum reservoir (Fench summary) RAIRO model; Math. Anal. Numer. 23 $5-51$.

Wadi A S, Dimian M F and Ibrahim F N 2014 Analytical solutions for one-dimensional advection-dispersion equation of the pollutant concentration; J. Earth Syst. Sci. 123(6) 1317-1324.

Wheatcraft S W and Tyler S W 1988 An explanation of scale dependent dispersivity in heterogeneous aquifer using concepts of fractal geometry; Water Resour. Res. 24(4) 566-578.

Yadav S K, Kumar A, Jaiswal D K and Kumar N 2011 Onedimensional unsteady solute transport along unsteady flow through inhomogeneous medium; J. Earth Syst. Sci. 120 205-213.

Yates S R 1990 An analytical solution for one-dimensional transport in heterogeneous porous media; Water Resour. Res. 26 2331-2338.

You K and Zhan H 2013 New solutions for solute transport in a finite column with distance dependent dispersivities and time-dependent solute sources; $J$. Hydrol. 487 87-97.

Zech A, Attinger S, Cvetkovic V, Dagan G, Dietrich P, Fiori A, Rubin Y and Teutsch G 2015 Is unique scaling of aquifer macrodispersivity supported by field data?; Water Resour. Res. 51 7662-7679. 